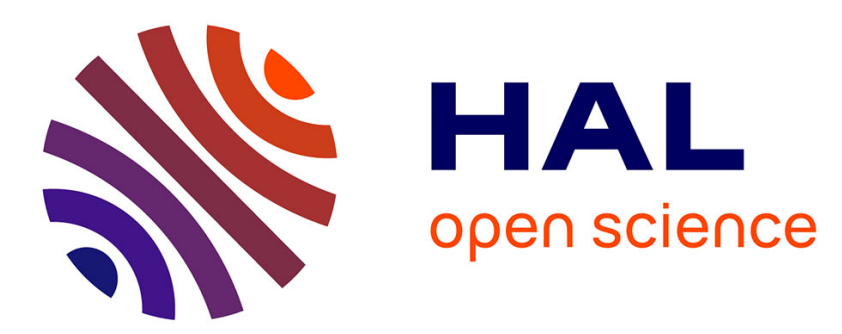

\title{
Contribution of heterogeneous strain field measurements and boundary conditions modelling in inverse identification of material parameters
}

\author{
Thomas Pottier, Franck Toussaint, Pierre Vacher
}

\section{- To cite this version:}

Thomas Pottier, Franck Toussaint, Pierre Vacher. Contribution of heterogeneous strain field measurements and boundary conditions modelling in inverse identification of material parameters. European Journal of Mechanics - A/Solids, 2011, 30 (3), p. 373-382. hal-00596629

\section{HAL Id: hal-00596629 \\ https://hal.science/hal-00596629}

Submitted on 24 Sep 2021

HAL is a multi-disciplinary open access archive for the deposit and dissemination of scientific research documents, whether they are published or not. The documents may come from teaching and research institutions in France or abroad, or from public or private research centers.
L'archive ouverte pluridisciplinaire $\mathbf{H A L}$, est destinée au dépôt et à la diffusion de documents scientifiques de niveau recherche, publiés ou non, émanant des établissements d'enseignement et de recherche français ou étrangers, des laboratoires publics ou privés. 


\title{
Contribution of heterogeneous strain field measurements and boundary conditions modelling in inverse identification of material parameters
}

\author{
T. Pottier ${ }^{a *}$, F. Toussaint ${ }^{a}$, P. Vacher ${ }^{a}$
}

${ }^{a}$ Laboratoire SYMME - Polytech'Savoie BP 80439, 74944 Annecy le Vieux Cedex, France.

\author{
KEYWORDS \\ Full-field measurement \\ Inverse problem \\ Plastic anisotropy \\ Measurement noise \\ Mechanical characterization.
}

\begin{abstract}
The present paper aims at applying the Finite Element Updating inverse method to several sample geometries by the means of Digital Image Correlation. The full-field data are experimentally obtained from three geometries exhibiting increasing strain fields heterogeneities. For each test, a Finite Element model is built and boundary conditions are duplicated from the measured displacements at the sample borders. Field comparisons are performed at several time steps until fracture occurs and a Levenberg-Marquardt method is used to solve the optimization problem. Six parameters of an anisotropic elastic-plastic constitutive model are identified and validated through the simulation of a deep-drawing forming operation. Results show that identification quality is improved when heterogeneous strain fields are used.
\end{abstract}

\section{Introduction}

Facing the increasing number of new materials and the difficulties of identifying constitutive models that predict properly the material behaviour during the forming process, industrials have induced in the early 90's some scientific research that challenges the usual way of identifying material models. This former approach consists in fitting analytical model with the experimental behavior (true stress/true strain curve). Basically, tests are led on standard geometries to isolate one material behaviour from the others (e.g. a tensile test is assumed to exhibit tensile behaviour only), then hypothesis concerning the strain field homogeneity are made and lead to identify the corresponding constitutive parameter. Once these constitutive parameters are determined, the Finite Element (FE) analysis is used to retrieve the stress/strain/displacement fields, assuming that both geometry and boundary conditions are known. This identification procedure exhibits two main disadvantages: the numbers of tests may increase significantly when complex models are in use and the hypothesis of homogeneous strain fields is neither always obvious nor easy to satisfy. On the other hand, the so-called inverse approach proposes to determine constitutive parameters from considering heterogeneous strain/displacement fields. In this case, geometry and boundary conditions are known and so is the resulting displacement field. Therefore, the only constitutive parameters remain unknown. The chosen constitutive equations are, of course, assumed to be relevant.

As Summarized by [15] and [3], several different methods have been developed to obtain the solution of such inverse problems. Indeed five approaches lead to retrieve the constitutive parameters when the stain homogeneity hypothesis does not hold. Among these, the constitutive equation gap

\footnotetext{
*Tel. +33 (0)563493048
}

e-mail: thomas.pottier@mines-albi.fr

www.institut-clement-ader.org method [25, 26], the equilibrium gap method [6, 9], the virtual fields method [14, 39], the reciprocity gap method [21] and the finite element model updating (FEU) technique [23, 35] are currently used for identification purpose. In the present paper the FEU approach has been chosen.

The FEU method provides constitutive parameters iteratively by comparing the measured data with the numerical ones while parameters vary. The experiment is accurately simulated in a FE model and the calculation is run with an initial guess of the parameters values. The obtained displacement fields are then compared to the measured ones, a cost function is built and leads to a new set of parameters and so forth, until convergence is reached. The inverse methods applied to parameters identification such as defined first by [23] have known massive improvements in the last decade. Indeed, the FEU approach is now broadly used. Several authors have applied FEU to identify material parameters using so-called global measurements, such as reaction force of the whole sample, as the material response. For instance, [12] have identified 5 parameters of an elastic-plastic constitutive law of aluminum alloy using tensile tests in a first approach and a deep drawing test in a second one. Both results were very close and authors conclude that this approach is an alternative to the classical identification method. Moreover, the experiments led by [45] have successfully identified both elastic and elastic-plastic parameters using flexion tests. The torque was used as the material response and the experimental and numerical curvature/torque curves were compared for several parameters sets until fitting was reached. Both of these studies have provided satisfactory results but none of them was using full-field measurement as experimental data. Meanwhile, [34] has developed a similar approach with the use of full-field measurements. Shear-like tensile tests were 
performed and displacement fields were measured. A cost function was built considering global reaction force and displacement fields as the material responses. For the sake of simplicity, only the early beginning of the plastic process was taken into account and neither high strain nor necking was considered. Recently this work has been completed by [22]. This latter study deals with through necking analysis of basic tensile specimens with side notches. This geometrical singularity ensures the necking to occur at the center of the sample and improves the heterogeneity of the strain fields. The identification of two elastic-plastic model is achieved but no conclusion is drawn upon the contribution of considering the whole deformation process instead of the early stages of it. Finally the work of [29] and [7] also has to be noticed. As an improvement to the works presented above, it introduced the use of a significantly more heterogeneous tests thanks to bi-axial tensile tests. Cruciform samples are then used to identify elastic-orthotropic parameters.

The present paper aims to show that identification quality improves and the required number of experiments decreases as the heterogeneity of strain fields increases. For this purpose, the FEU inverse method is applied to several sample geometries, using Digital Image Correlation data. Three geometries with increasing strain field heterogeneity are tested, and provide the parameter set of an anisotropic elastic-plastic model. For the sake of comparison, the same identification procedure is used for the three geometries. Boundary conditions of the FE model are strictly applied from the experiment and allows the FE model to fit perfectly the experimental deformation process. Thus, the identification calculations are run again using virtually noised experimental data in order to exhibit and quantify the sensitivity of the identification method to measurement noise. Finally, validation tests are led on a deep-drawing operation and results are used to compare the quality of the obtained parameter sets.

\section{Experimental Setup}

\section{Material and samples geometries}

All the samples under investigation are made of Commercially Pure titanium T40, the limits of the chemical composition of the material are given in Table 1 . The average grain size has been estimated to $6.4 \mu \mathrm{m}$. This material, broadly used in aeronautics and biomedical domains, consists of hot-rolled sheets where the $0^{\circ}$ direction is chosen to fit the rolling direction. As a consequence, $90^{\circ}$ is the transverse direction of the rolled sheet. Sheet thickness is $0.5 \mathrm{~mm}$.

As shown in Fig. 1, three sample shapes were used. A basic tensile sample (no.1) as used by [32], [13] and many others, will first be investigated. Then, the sample geometry is added a hole (no.2) as suggested by [28] and finally a shearlike tensile sample (no.3) will be studied. This latter sample, originally proposed by [34], is expected to exhibit both tensile and shear behaviours.

\section{Full-field measurements}

In the present study, The Digital Image Correlation method is used to assess the local displacements at the sample surface with $7 \mathrm{D}$ correlation software [43]. The experimental setup is shown in Fig. 2a.

Tests are led on a $5 k N$ INSTRON tensile device. The samples undergo a prescribed displacement speed of $5 \cdot 10^{-5} \mathrm{~m} \cdot \mathrm{s}^{-1}$ (corresponding to an approximate strain rate $\dot{\varepsilon} \approx 1 \cdot 10^{-3} s^{-1}$ ) until their ruin. Samples are speckled using black and white mate painting sprays. Digital images are recorded during the deformation process at a frequency of $0.25 \mathrm{~Hz}$ using a Nikon D200 camera (focal distance $105 \mathrm{~mm}$ ). The image resolution is set to $2592 \times 3872$ grey level pixels and stored as 8 bit (Fig. 2b). Moreover, axial reaction force is recorded every $10 \mu s$ all along the test. For each test, the initial image is split in square elements that create a virtual grid upon the sample surface. The resolution of this grid (extensometric base) is set to $16 \times 16$ pixels (Fig. $2 \mathrm{~b}$ ), corresponding to $0.32 \times 0.32 \mathrm{~mm}^{2}$. The correlation process consists in looking for the most probable deformed pattern in the neighbourhood of each node of this grid in terms of grey level. The displacement fields of each element are then assessed by the means of a bi-linear interpolation. Finally, the displacement at image $n$ is assessed by correlating the $n^{\text {th }}$ image with the initial/undeformed image. The correlation parameter used in the present study are summarized in Table 2.

The assessment of DIC technique uncertainties remains a topical issue [5], [40] and [22]. Several parameters may have influence on the measurement noise such as lighting, strain gradients, off-plain displacements, numerical noise of the camera and of course the quality of the speckle [37]. However, the works of [5] and [8] (performed with the correlation parameter presented in Table 2) state that the order of magnitude of the DIC measurement noise remains inbetween 0.01 and 0.1 pixel.

\section{Identification Scheme}

\section{Inverse flowchart}

In the present study, the FEU solving flowchart (Fig. 3) is chosen and applied to parameters identification . Experiments are led and post-processed using 7D correlation software then data are imported into Matlab. A finite element model of the sample is designed and meshed using Abaqus-Explicit. This latter FE model is multiply solved for several sets of material parameters using the in situ prescribed conditions obtained by the means of DIC. Then, the FE calculation outputs are post-processed using Matlab in order to build the cost function that leads to the solution of the global identification problem.

\section{Cost-function}

Since, the comparison between experimental and numerical fields has to be computed, the relevant point is the manner of writing this comparison down. Several authors such as [34], [22] and [7] are using a weighted least square formulated cost-function. The introduction of a weighting matrix has basically two main kinds of formulation. First, it could be made up of the experimental errors and make the cost function a statistical estimator as did by [28] or [34]. Each nodal response is then compared with its experimental equivalent and divided by the estimated error at the considered material point. As a consequence, the more reliable a measure is, the 
Table 1: Chemical composition limits of commerciallypure grade 2 titanium

\begin{tabular}{ccccccc} 
Element & $\mathrm{Ti}$ & $\mathrm{C}$ & $\mathrm{Fe}$ & $\mathrm{N}$ & $\mathrm{O}$ & $\mathrm{H}$ \\
Weight $(\%)$ & Bal. & 0.10 & 0.30 & 0.03 & 0.25 & 0.015 \\
\hline
\end{tabular}

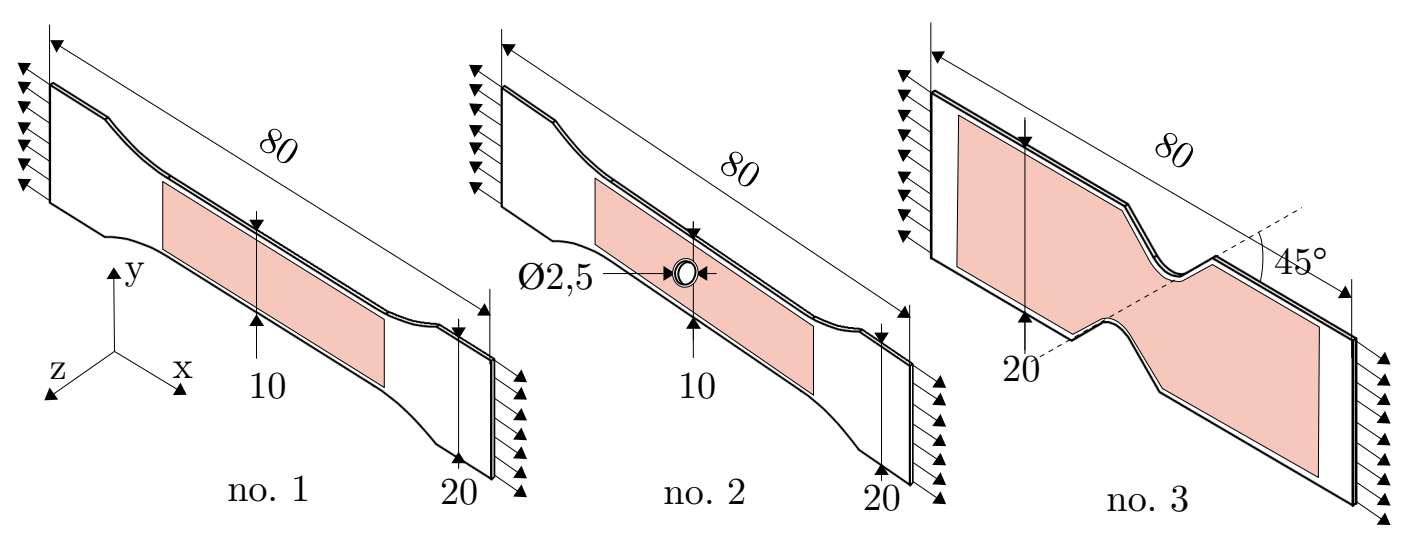

Figure 1: Investigated geometries and DIC analysis zones (dimensions in mm).

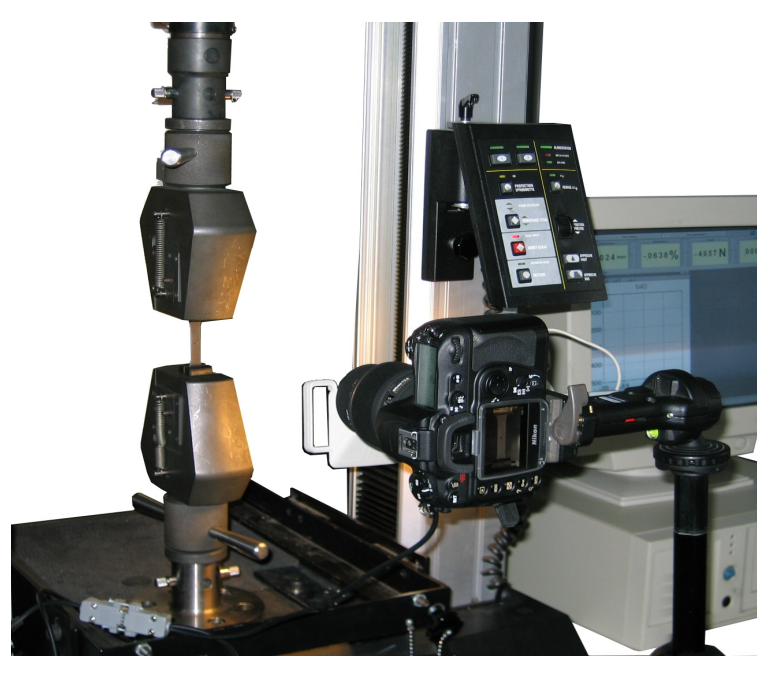

(a)

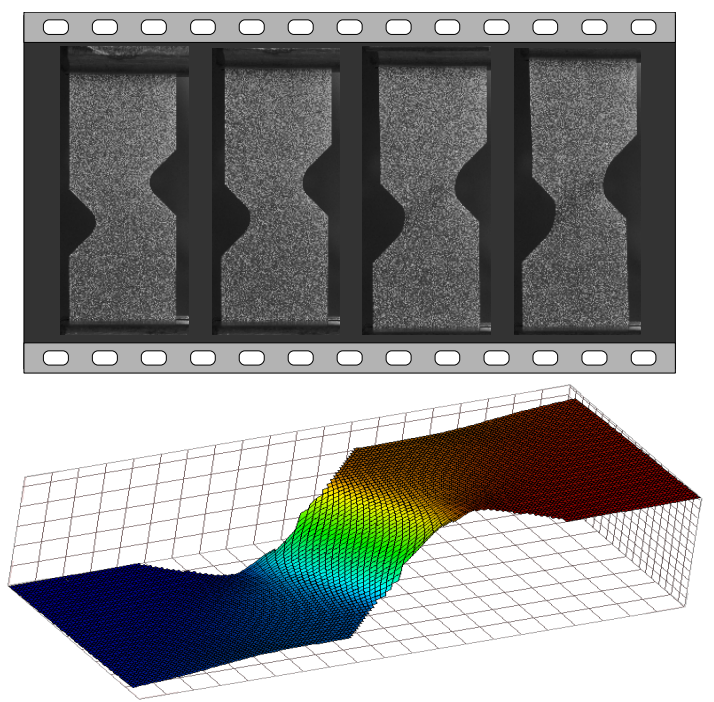

(b)

Figure 2: (a) Experimental setup. (b) $2592 \times 3872$ grey level images from Nikon D200 camera and longitudinal displacement field calculated by $7 \mathrm{D}$ correlation software.

Table 2: Main parameters of the Digital Image Correlation

\begin{tabular}{cccc}
\hline $\begin{array}{c}\text { Grid } \\
\text { size }\end{array}$ & $\begin{array}{c}\text { pattern } \\
\text { size }\end{array}$ & $\begin{array}{c}\text { grey level } \\
\text { interpolation }\end{array}$ & $\begin{array}{c}\text { displacement fields } \\
\text { interpolation }\end{array}$ \\
\hline $16 \times 16$ & $16 \times 16$ & bicubic & bilinear \\
\hline
\end{tabular}




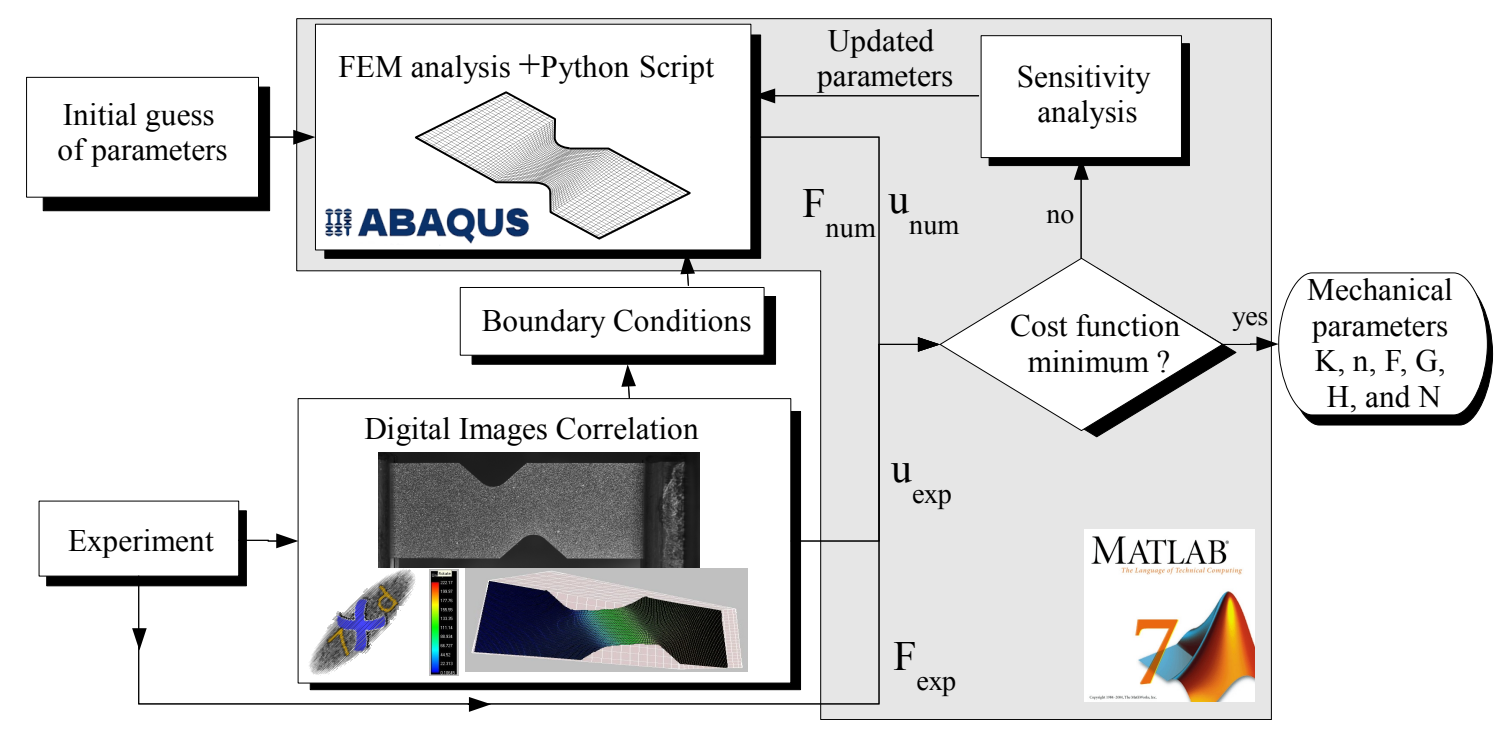

Figure 3: Iterative solving flowchart of the inverse problem.

more weight it has on the identification solution. Another approach consists in considering the weighting matrix as a way to give each material point the same weight. This solution consists in dividing each local residual by the measured response at this point. Such a formulation, used by [38], [7] and [24], also allows the use, in the same cost-function, of several kind of quantities exhibiting miscellaneous orders of magnitude. This latter approach is chosen in the present study.

Moreover, the residual quantity can be made of force and/or displacement data [23]. As mentioned in [3], considering both force and displacement in the cost function formulation lead to a better suited solution when strain concentration is involved (geometries no.2 and no.3 for instance). Therefore, in the present study, force and displacement data has been mixed using a weighting matrix in order to obtain a dimensionless formulation of the cost function. Indeed, longitudinal and transversal displacements are added to the global reaction force residual and the local difference between these three measured data and the calculated values is the function to be minimized. This cost-function can be written as follows:

$$
\begin{aligned}
f(p)= & \frac{1}{2}\left[\sum _ { i , j , k = 1 } ^ { N _ { t } , N _ { s } , N _ { n } } \left[\left(\frac{u_{x, j k}^{e x p}(p)-u_{x, j k}^{n u m}}{\max _{j, k}\left(u_{x}^{e x p}\right)}\right)^{2}+\right.\right. \\
& \left.\left.\left(\frac{u_{y, j k}^{e x p}(p)-u_{y, j k}^{n u m}}{\max _{j, k}\left(u_{y}^{e x p}\right)}\right)^{2}+N_{n}\left(\frac{F_{j}^{e x p}(p)-F_{j}^{n u m}}{\max _{j}\left(F^{e x p}\right)}\right)^{2}\right]_{i}\right]^{\frac{1}{2}} .
\end{aligned}
$$

Where $p$ is the unknown parameter column, $u_{x}$ is the displacement along the $x$ axis (longitudinal axis),$u_{y}$ the displacement along the $y$ axis (transverse axis) and $F$ is the global reaction force of the sample. The scalar $N_{n}$ is the number of nodes in the finite element mesh, $N_{s}$ is the number of time steps taken into account, and $N_{t}$ is the number of tests that are considered. Finally, $\max _{j, k}\left(u_{x}^{e x p}\right), \max _{j, k}\left(u_{y}^{e x p}\right)$, and $\max _{j}\left(F^{e x p}\right)$ are respectively the nodes greatest values over one step-time of the longitudinal and transversal displacements and the global reaction force. Hence, the optimal set of parameters must satisfy three conditions: matching the two displacement fields and the global force response (which is actually not a field response but a punctual one). The next section discuss the algorithm used to solve this optimization problem.

\section{Optimization algorithm}

Various numerical algorithms are available to solve non-linear least square problems represented by a cost function such as Eq. (1). Here, a Levenberg-Marquardt (LM) [30] and [33] method is used to minimize the cost-function. This method is known to have several advantages among which can be singled out that it does not require the assessment of the Hessian matrix and it's thus very easy to implement. Let's recall that the updating equation of a LM method is:

$$
\left(J^{T} J+\lambda_{L M} I\right)\left(p^{(k+1)}-p^{(k)}\right)=J^{T} r(p)
$$

Where $r(p)$ is the residual column constituted by the three $\left(u_{x}, u_{y}\right.$ and $\left.F\right)$ relative field differences at each node. Matrix $J$ is the Jacobian matrix that is often called sensitivity matrix. This latter matrix is assessed by computing the partial derivative, among each constitutive parameter, of every single system response (see section Sensitivity analysis). Finally, $\lambda_{L M}$ is a damping parameter that has been introduced by [30] to stabilize the Gauss-Newton method in the vicinity of the optimum. The experimental errors on the measured responses (displacement fields and global reaction forces) lead to the inexistence of an optimal parameters set. In other words, updating the constitutive parameters is not sufficient to obtain numerically the exact observed behaviour. Hence, the optimization problem is ill-posed [28] and a basic GaussNewton's method may become very unstable around the optimum. The addition of the $\lambda_{L M}$ parameter improves the robustness of the algorithm near the optimum. As proposed by [31], the value of $\lambda_{L M}$ is assessed as follows:

$$
\lambda_{L M}=\theta\|r(p)\|+(1-\theta)\left\|J^{T} r(p)\right\|
$$

Where $\theta \in[0,1]$ is a real parameter that must be set $a$ priori and that rules both accuracy and convergence rate of the algorithm. In the present work $\theta=8 \cdot 10^{6}$. 


\section{Sensitivity analysis}

The so-called sensitivity analysis consists in assessing the terms of the Jacobian matrix. As presented by [41], sensitivity assessment is a major issue in parameter identification. For this purpose a broadly used approach consists in computing sensitivities by the means of finite differences. Even though the calculation time is high, this approach is easy and fast to implement. Solving each iteration require only $k+1$ calculations if a forward (or backward) scheme is chosen. For the sake of calculation time, a forward finite difference scheme is used in this work, therefore sensitivities are assessed by the following assumption:

$$
J_{i, j}=\frac{\partial r_{i}(p)}{\partial p_{j}} \approx \frac{r_{i}(p)-r_{i}\left(p+\Delta p_{j}\right)}{2 \Delta p_{j}}
$$

Where $j$ is the number of constitutive parameters to be identified and $i$ is the number of system response, $\Delta p_{j}$ is the relative perturbation on the $j^{\text {th }}$ parameter. The choice of this perturbation $\Delta p_{j}$ remains a controversial topic. Indeed, the above equation exhibits a truncation error of $\Delta p_{j}^{2}$ that tends toward zero when the perturbation decreases. On the other hand, it has to be noticed that the decreasing of the perturbation value increases the weight of the round-off error, therefore the value of $\Delta p_{j}$ results from a compromise between these two errors. In the present study the perturbation value is set to $\Delta p_{j}=0.01 \times p_{j}$.

\section{Material and Numerical Model}

\section{Numerical model}

The numerical model, developed using Abaqus, duplicates the experiment in terms of geometry and boundary conditions. The geometry is measured on the samples, modeled and meshed using quadrangular shell elements (the number of nodes varies from 1458 to 2201 depending on the considered geometry). The obtained meshes fit the three geometries under investigation. As shown in Fig. 4, boundaries conditions, are measured from the experiments using DIC and the obtained displacements are prescribed at each node of the upper and lower bounds of the mesh $[1,44,10]$. Both longitudinal and transverse displacements are imposed. However, the data measured from the DIC may not properly fit the chosen nodes of the FE mesh. In other words, the displacement data may be obtained at a material point that does not match a node of the FE mesh because DIC grid and FE mesh are different. Therefore, the DIC displacement fields are interpolated at each node of the FE mesh so that the fields comparisons (needed for the cost function assessment) can be computed at the same material points (Fig. 4). This projection is processed using bi-linear interpolation shape functions.

\section{Constitutive equations}

The presented identification scheme can basically be applied to any kind of constitutive model. In the present study, a classical anisotropic elastic-plastic model is considered. The elastic strain is here defined by the Hooke's formulation, where $E$ and $\nu$, respectively the Young's modulus and the Poisson's ratio are previously identified $(E=111.8 \mathrm{MPa}$ and $\nu=0.34)$. A large strain framework is used and $\boldsymbol{\sigma}$ is the Cauchy stress tensor, $\mathbb{E}$ is the Green-Lagrange strain tensor. The generalized standard material formalism provides the plastic strain, that can be written as follows:

$$
d \mathbb{E}_{p}=d \lambda \frac{\partial f(\boldsymbol{\sigma})}{\partial \boldsymbol{\sigma}}, \quad d \lambda \geq 0
$$

where $d \lambda$ is a non-negative scalar. This latter formulation allows the introduction of a yield surface and therefore a yield criterion can be written:

$$
f(\boldsymbol{\sigma})=\mathcal{J}(\boldsymbol{\sigma})-R-\sigma_{y}
$$

Where $R$ is the current yield stress, $\sigma_{y}=368.0 \mathrm{MPa}$ is the initial yield stress and $\mathcal{J}(\boldsymbol{\sigma})$ is the equivalent plastic stress. In the present study, an orthotropic material behaviour will be assumed and the Hill's 1948 [19] criterion is used. This criterion is commonly used for sheet metal anisotropic behaviour and it provides an expression of the equivalent stress. In the case of plane stress, which is assumed here, the Hill's criterion gives:

$$
\mathcal{J}(\boldsymbol{\sigma})=\sqrt{F \sigma_{22}^{2}+G \sigma_{11}^{2}+H\left(\sigma_{11}^{2}-\sigma_{22}^{2}\right)+2 N \sigma_{12}^{2}}
$$

As a consequence, the description of material anisotropy only requires four parameters to be identified. As presented by [20], in practice the use of the Lankford's coefficients is easier. The present study will focus on the determination of these coefficients that are defined in section Standard identification. Moreover, the material hardening described by $R$ has to be considered. Several models have been developed in the last decades in order to take into account those effects [16]. For the sake of simplicity, the Ludwik's flow rule is chosen, it is both easy to implement and to identify because only two parameters are needed. The writing of such a flow rule is recalled as:

$$
R=K\left(\mathbb{E}_{p}^{e q}\right)^{n}
$$

Where, $\mathbb{E}_{p}^{e q}$ is the equivalent plastic strain. $K$ and $n$ are two parameters to be identified. Finally, an anisotropic elastic-plastic model is in use and the present identification study deals with only six parameters.

\section{Results and Validation}

\section{Standard identification}

In order to offer a comparison criterion to the results obtained by the means of inverse method, a standard identification has been proceeded. As presented by [42], this approach is based on several assumptions and provides a quick assessment of material constitutive parameters. The strain fields obtained using DIC are used to localize the necking area of the sample. Therefore, only the local strain data are considered and the volume conservation assumption is made to allow the assessment of the width at the necking location and the troughthickness Hencky's strain $\left(\varepsilon_{I I I}^{p}\right)[17]$. Thus, the true stress can be computed and plotted versus the plastic equivalent strain (Fig. 5a). Hence, the hardening curve is fitted using a least-square routine which leads to identify both $K$ and $n$ : the Ludwik's parameters.

Anisotropic behaviour is also identified and the Hill's parameters are assessed through the Lankford's coefficients. Let's recall that those coefficients are defined as follows: 

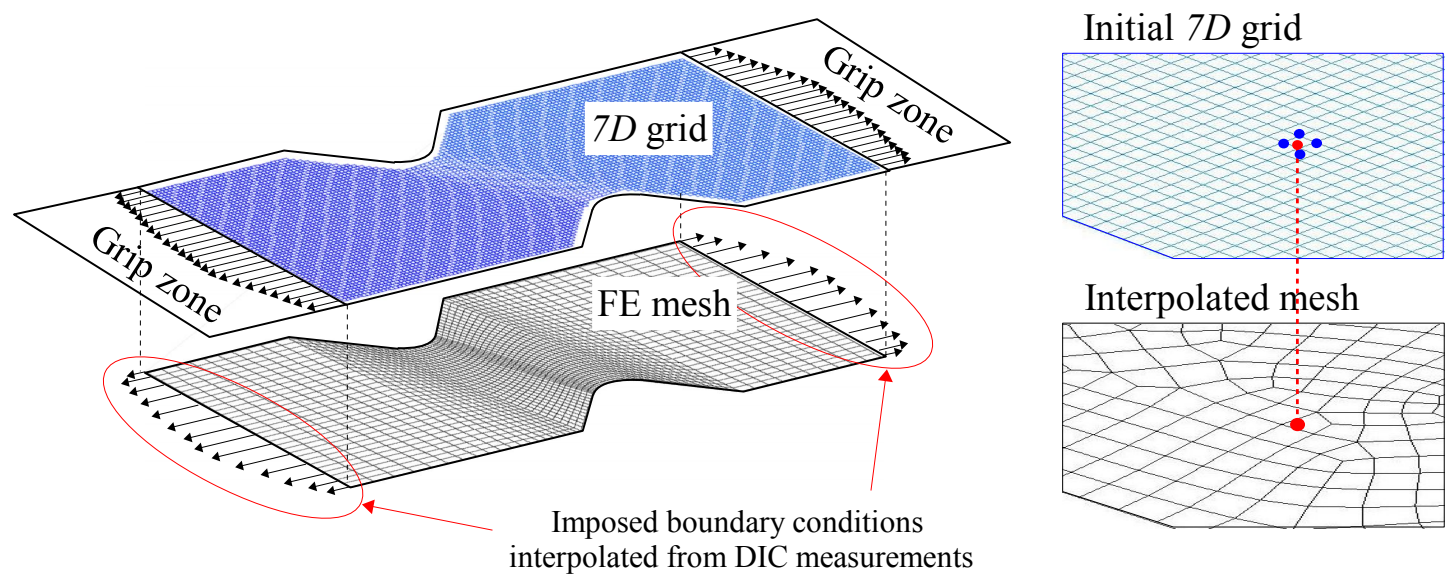

Interpolated mesh

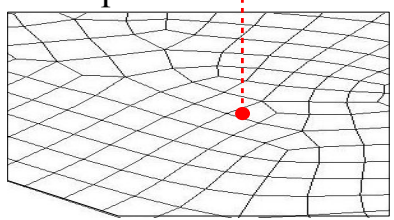

Figure 4: Experimental field measurements and boundary conditions were interpolated from the DIC analysis.
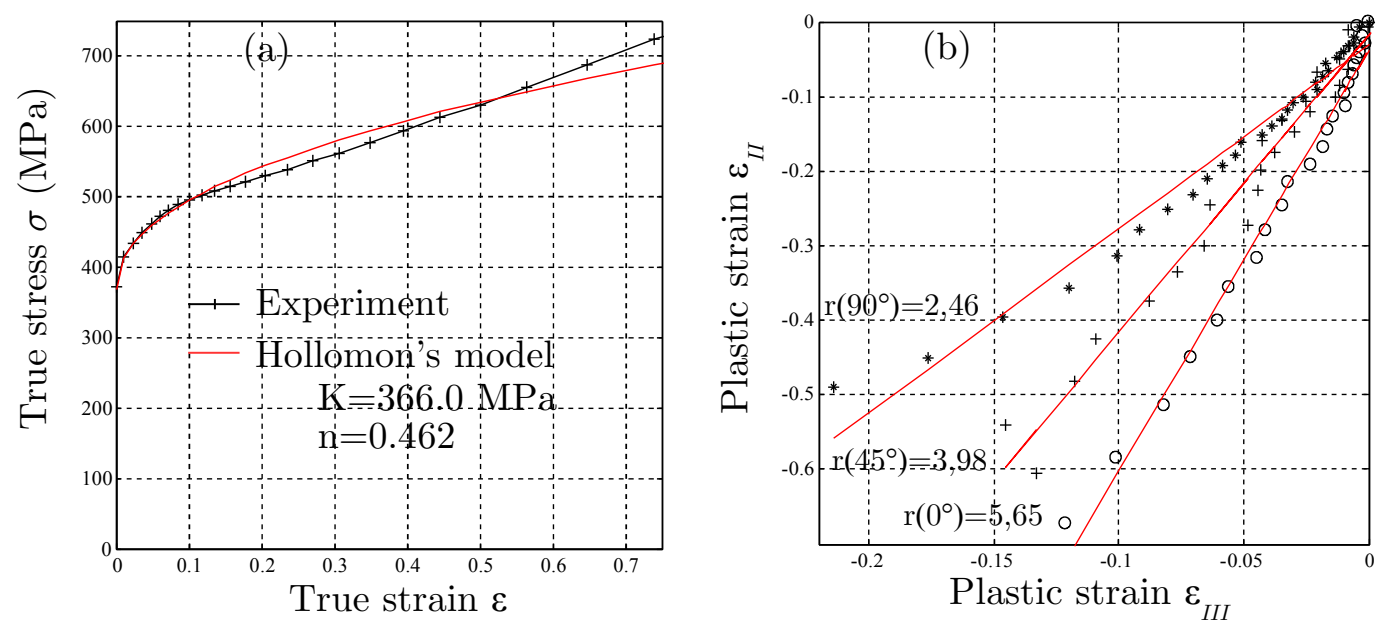

Figure 5: Standard identification of the five investigated parameters. (a) Ludwik's parameters. (b) Lankford's coefficients. 


$$
r_{\theta}=\frac{d \varepsilon_{I I I}^{p}}{d \varepsilon_{I I}^{p}}
$$

Where $\theta$ is the angle between the rolling and the applied tensile direction. This ratio of the transverse plastic strain rate to the thickness plastic strain rate is experimentally represented by the slope of the measured strains in the tensile tests led at $0^{\circ}, 45^{\circ}$ and $90^{\circ}$ from the rolling direction. Their respective least-square lines are also plotted in Fig. 5b. Finally the three Lankford's parameters: $r_{0}, r_{45}$ and $r_{90}$ are identified and lead to assess the four Hill's parameters that are summarized in Table 3.

\section{Inverse identification}

For the sake of calculation time, the parameters identification is processed considering only a restricted number of time steps arbitrary chosen over the hardening curve. This number $N_{s}$ varies inbetween 5 and 7 and they can be seen as markers in Fig. 6.

For every geometry, three tests are considered $\left(N_{t}=3\right)$ : at $0^{\circ}, 45^{\circ}$ and $90^{\circ}$ except for geometry no. 3 where two identification procedures are run: the first with $N_{t}=3$ and the second with $N_{t}=2$. This aims to show that when strain fields heterogeneity increases, the number of required tests may be decreased without impairing the identification quality.

In order to impose the necking to occur at the same location in experiments as in the FE analysis of geometry no.1, a small width default $(0.05 \mathrm{~mm}$ on each side) is introduced on the initial mesh. No special treatment or assumption is made for the other analysis. The initial hardening parameters are arbitrarily chosen equal to $K=550 \mathrm{MPa}$ and $n=0.3$, the Hill's coefficients are chosen to initially match the isotropic case: $F=G=H=0.5$ and $N=1.5$. The identified parameters are summarized in Table 3 . and compared to those identified using the standard method.

Table 3 shows that the proposed inverse method is able to decrease the number of tests needed for identification and thus the experimental work. Indeed parameters sets no.3-a and no.3-b are very similar and can be considered as equal (denoted set no.3).

As shown in Fig. 6, the four obtained sets (sets no.1, no.2, no.3 and the standard identification set) are used to predict the global reaction force of three tests with the different geometries. It can be seen that the set no. 3 is the most able to provide a good force prediction regardless of geometry. The fitting of the set no.1 is very poor. Indeed this set, identified in a pure tensile load case, appears to be unable to predict the global reaction force when other kinds of strain fields are involved (e.g. test no.3). This emphasizes the improvement of parameter identification when heterogeneous strain fields are used.

Moreover, Fig. 7 shows the identified resulting displacement fields versus the experimental ones for set no.3, the residual values are also plotted for both longitudinal and transverse displacement fields. As can be seen, the optimization process leads to the fitting of experimental and numerical responses.

\section{Experimental noise sensitivity}

Since the parameter set is identified, the predicting capability of it may be investigated. As pointed in many former studies $[11,18,35,29,2,4,36,39]$, investigating the noise sensitivity if the identification procedure is a good way to evaluate its stability. In practice, the noise of the measuring tools may be responsible for a high deviation of the obtained results. As said above, the accuracy of DIC method is limited and the measurement uncertainties assessment is a tough task. However some authors $[8,5]$ state that their magnitude remains inbetween 0.01 and 0.1 pixel. In the present study, displacement fields were artificially noised using a sample-wide random white noise of which magnitude varies. A random uniform noise is applied to both longitudinal and transverse displacement fields of the geometry no.3. The noise magnitude $A_{n}$ is set to several increasing values then the obtained parameter sets are compared. Results are summarized in Table 4 .

In addition, for the three noised sets, the predicted longitudinal displacement field is compared to the noiseless field. Fig. 8 shows the relative error $e\left(A_{i}\right)$ for the three noised sets versus the noiseless field such as:

$$
e\left(A_{i}\right)=\frac{U_{x}^{\text {num }}\left(p\left(A_{i}\right)\right)-U_{x}^{\text {num }}\left(p\left(A_{0}\right)\right)}{U_{x}^{\text {num }}\left(p\left(A_{0}\right)\right)}
$$

As can be noticed, the increasing of measurement noise quickly impairs the quality of the predicted displacement fields. This analysis shows that the FEU inverse method is obviously noise sensitive but also that for the assumed noise magnitude (between 0.01 and 0.1 pixel) the results dispersion remains acceptable (inferior to $3.9 \%$ ) and in the range of experimental uncertainties. Moreover, the obtained dispersion for 0.1 pixel matches other published results for inverse methods such as [39] and [22].

\section{Validation test}

In order to verify the quality of the identified parameters sets, validation tests are carried out. The three identified parameters sets are used to simulate the same deep-drawing operation. Results are compared to the experimental data in order to check which of the identified sets is the most able to reproduce the observed experimental behaviour.

On one hand a deep-drawing device (Fig. 9a) is used for the purpose of experimental validation. The elastic strength of both springs is previously identified and the device is operated using the same tensile machine as described above. Three circular blanks following the geometrical specification given in Fig. 9b are deep-drawn while operating force is recorded. Hence, the geometrical responses of the three obtained cups are measured. As shown on Fig. 9c the upper profile of the cups exhibit anisotropic horns and the magnitude and frequency of it are investigated and measured. A Coordinate Measuring Machine (CMM) is used to measure the height of 120 points evenly spread along the upper periphery. On the other hand, a FE model is developed to simulate the experimental deep drawing process. Solid elements are used to model the blank while die, blank-holder and punch are considered as analytical rigid surfaces. The

\footnotetext{
*2.66 GHz and RAM 0.98 Go
} 
Table 3: Parameters identified by inverse method.

Standard Geom. no.1 Geom. no.2 Geom. no.3-a Geom. no.3-b identification

\begin{tabular}{lccccc}
\hline$N_{s}$ & - & 6 & 4 & 6 & 6 \\
$N_{t}$ & 3 & 3 & 3 & 3 & 2 \\
Orientations & $0^{\circ}-45^{\circ}-90^{\circ}$ & $0^{\circ}-45^{\circ}-90^{\circ}$ & $0^{\circ}-45^{\circ}-90^{\circ}$ & $0^{\circ}-45^{\circ}-90^{\circ}$ & $0^{\circ}-45^{\circ}$ \\
\hline$K(M P a)$ & 366 & 349 & 267 & 284 & 280 \\
$n$ & 0.46 & 0.45 & 0.40 & 0.50 & 0.49 \\
$F$ & 0.30 & 0.31 & 0.21 & 0.16 & 0.16 \\
$G$ & 0.19 & 0.10 & 0.22 & 0.18 & 0.20 \\
$H$ & 0.81 & 0.90 & 0.78 & 0.82 & 0.78 \\
$N$ & 2.22 & 0.95 & 2.00 & 2.02 & 2.02 \\
\hline$f_{\text {end }} / f_{0}$ & - & $22.0 \%$ & $11.2 \%$ & $9.35 \%$ & $7.37 \%$ \\
$\mathrm{CPU}^{*}($ in $s)$ & - & 3858 & 5835 & 9523 & 5376 \\
\hline
\end{tabular}
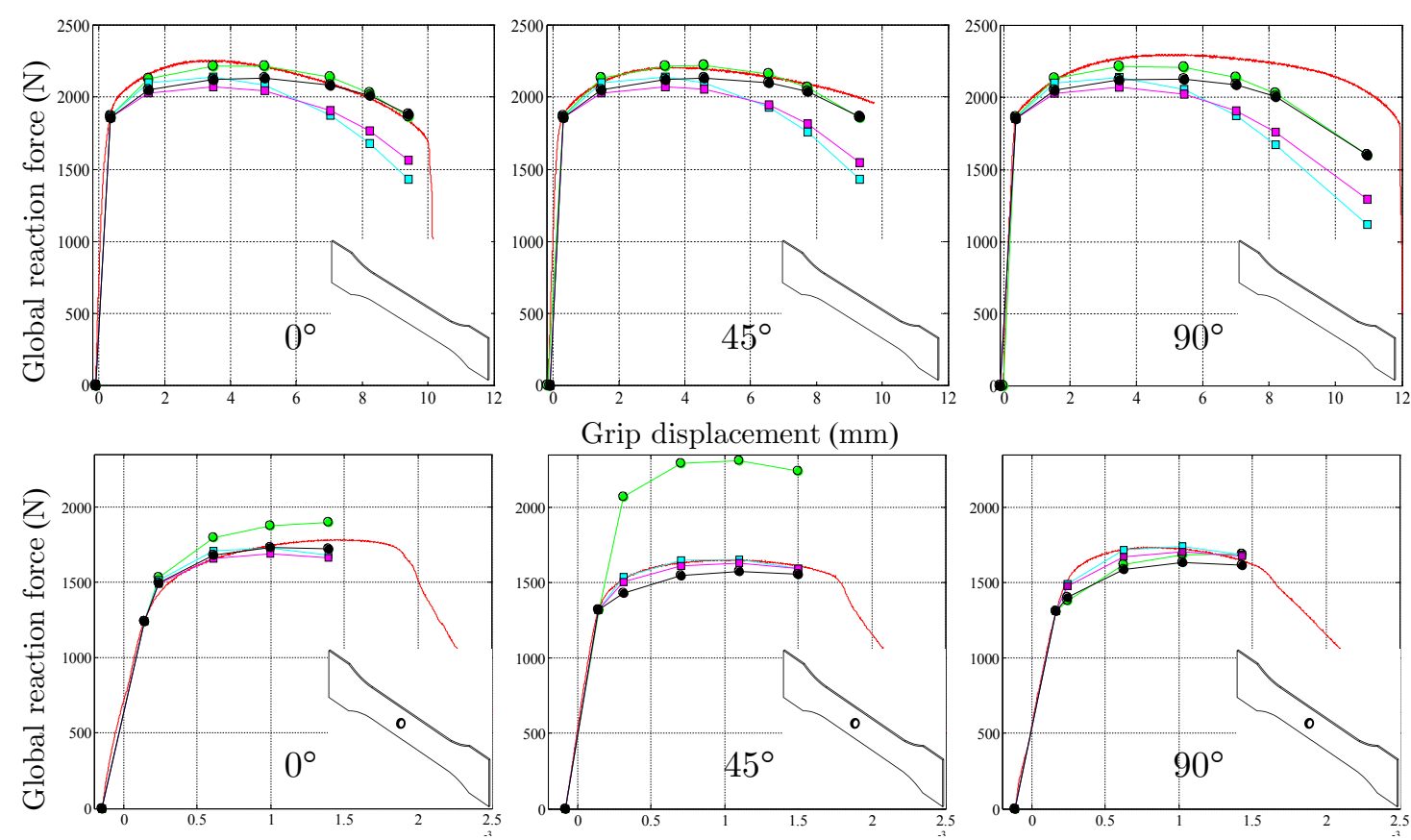

Grip displacement (mm)
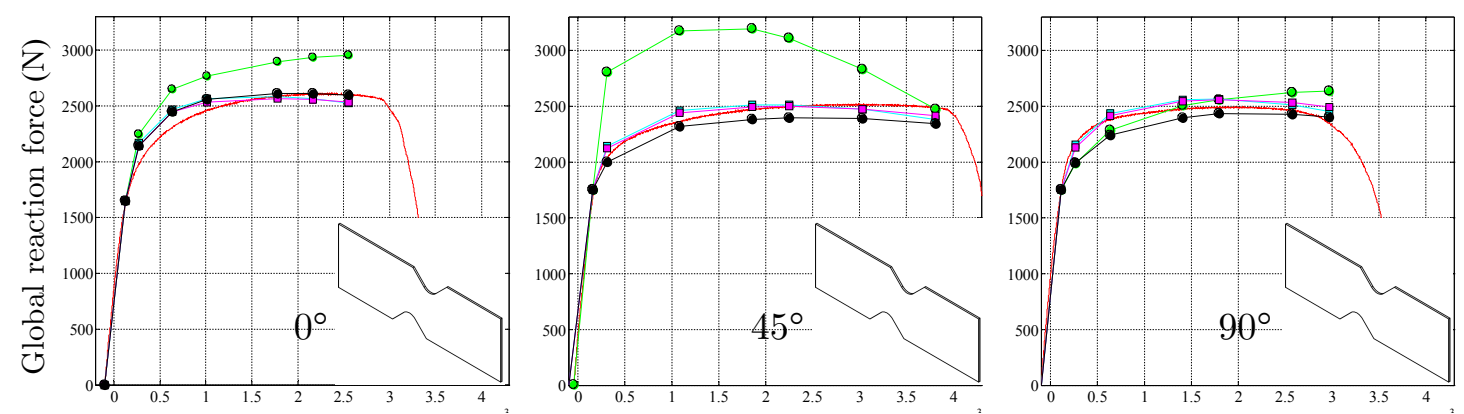

Grip displacement (mm)

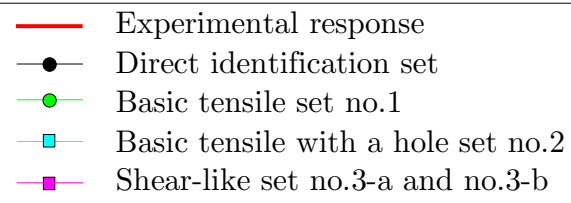

Figure 6: Comparison of the global force predictions of the four identified parameter sets. 


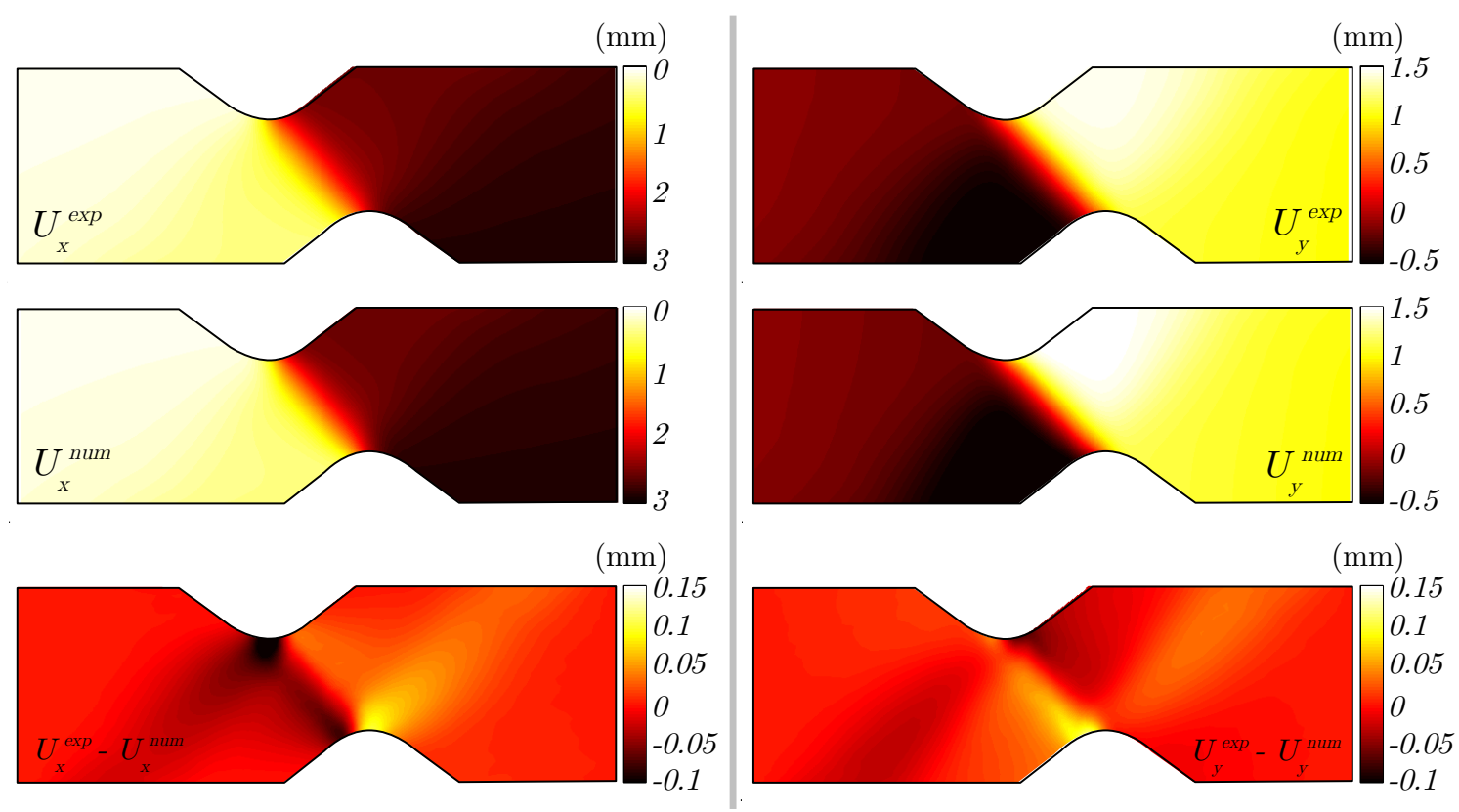

Figure 7: Experimental and identified displacement fields for the geometry no.3-a at $45^{\circ}$ ( $5^{\text {th }}$ time step). Residual fields show the zones of good and bad predictions.

Table 4: Parameters sensitivity to measurement noisefor shear-like geometry (no.3).

\begin{tabular}{lcccc}
\hline$A_{n}($ pixel $)$ & 0 & 0.01 & 0.1 & 1 \\
$A_{n}(\mu \mathrm{m})$ & 0 & 0.2 & 2 & 20 \\
\hline$K$ & 284 & 277 & 269 & 334 \\
$n$ & 0.50 & 0.48 & 0.49 & 0.48 \\
$F$ & 0.16 & 0.16 & 0.14 & 0.25 \\
$G$ & 0.18 & 0.19 & 0.15 & 0.26 \\
$H$ & 0.82 & 0.81 & 0.85 & 0.74 \\
$N$ & 2.02 & 2.01 & 2.01 & 2.01 \\
\hline
\end{tabular}
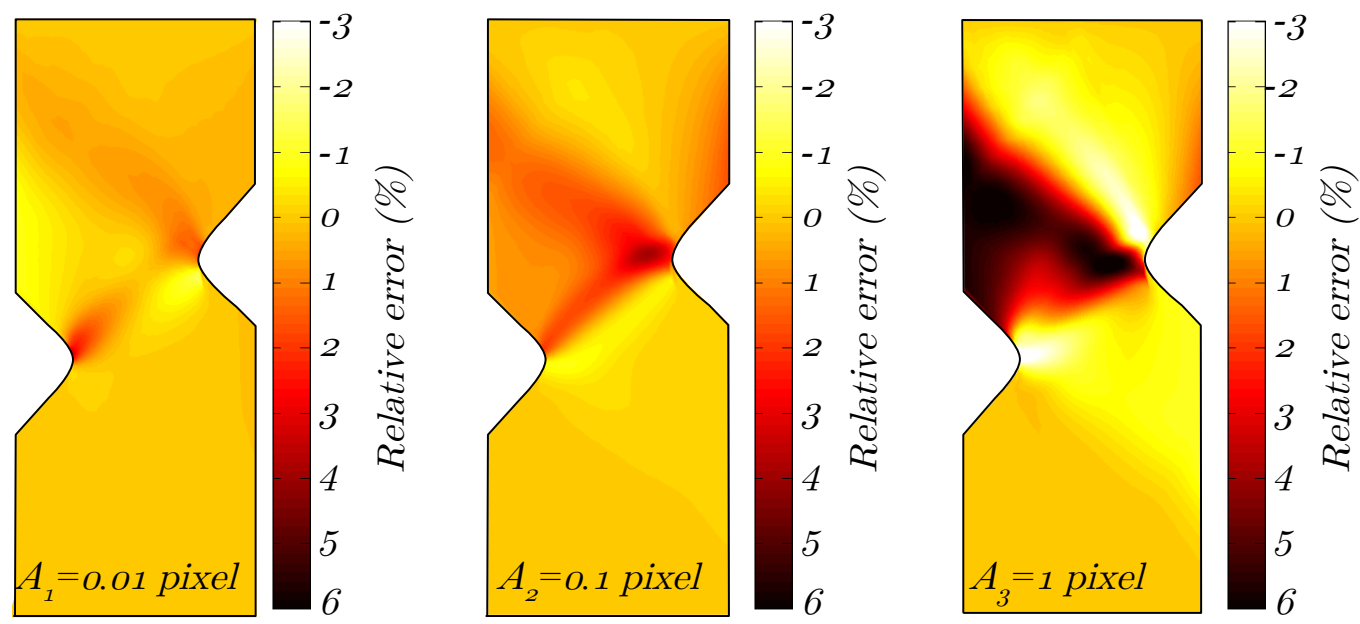

Figure 8: Relative error of the longitudinal displacement fields for the three noised set. 
(a)

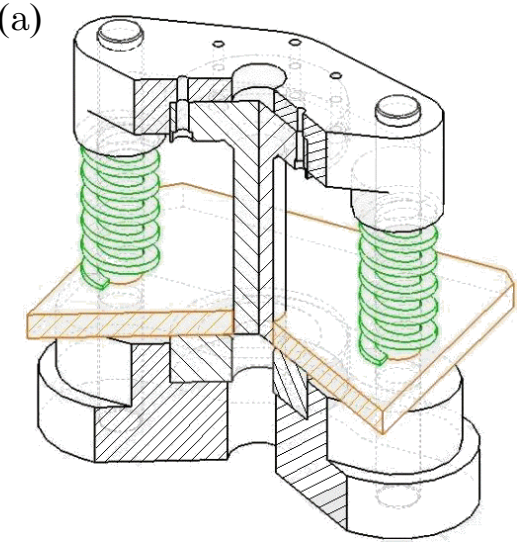

(b)

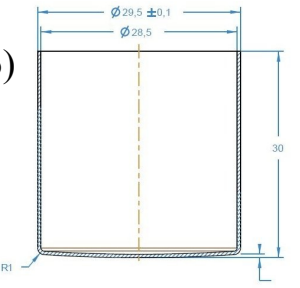

(c)

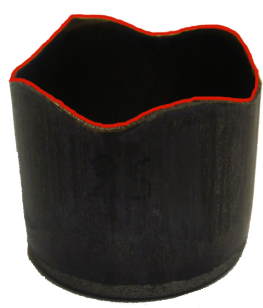

Figure 9: (a) Deep-drawing device, (b) cup geometry and (c) experimental cup shape.

applied load on the blank-holder is picked from the experimental measurements. The friction coefficient is set to 0.2 in agreement with former results [27]. Finally the identified parameter sets are successively used. Then, the upper profile is compared to the experimental one (Fig. 10). The simulations with inverse parameter sets exhibit very scattered anisotropic responses, indeed eventhough geometries no. 2 and no. 3 show a good fitting with the experimental measurements, the response of geometry no. 1 is poor. This latter set predicts horns at the wrong place and with a bad magnitude. The simulation using the parameters identified using geometry no. 3 predicts more accurately the magnitude of the horns than geometry no. 2 and no.1.

The results of the validation tests, presented in Fig. 10 show that the quality of the shape prediction increases with the strain field heterogeneity. The numerical shape of the cup has a better fitting of the experimental shape when shearlike identified parameters are used. Eventhough the standard identification set remains the best for shape prediction, among the three inverse sets, the increasing of the strain heterogeneity in the sample lead to a more accurate identification of the material models.

\section{Conclusions}

In the present study, a FEU method has been used to identify the parameters of an anisotropic elastic-plastic model using full-field measurements. Three geometries that exhibit increasing strain heterogeneities have been investigated. Boundary conditions were picked at the borders of the DIC domain and strictly duplicated in the FE model. The results show that considering two experiments can provide the same results as three. Hence, the increasing of strain fields heterogeneity allows to perform less tests than homogeneous tests. Validation tests and comparisons between the identified parameter sets have shown that the ability of predicting the real deformation process is improved when the strain heterogeneity increases. However, the standard identification method remains the most able to predict material behaviour. This highlights the necessity of more heterogeneous tests when inverse methods are used. Finally, experimental data were noised in the range of known admissible DIC uncertainties. The obtained dispersions remain in the range of other published works. The presented identification tool provides satisfying results with kinematics data but cannot identify fully coupled thermal-mechanical models so far. Thus, further works will focus on considering both kinematic and thermal fields but also on the ways to experimentally obtain heterogeneous strain fields

\section{References}

[1] J. Abanto-Bueno and J. Lambros. Experimental determination of cohesive failure properties of a photodegradable copolymer. Experimental Mechanics, 45(2):144-152, 2005.

[2] F. Amiot, F. Hild, and J-P. Roger. Identification of elastic property and loading fields from full-field displacement measurements. International Journal of Solids and Structures, 44:2863-2887, 2007.

[3] S. Avril, M. Bonnet, A.S. Bretelle, M. Grédiac, F. Hild, P. Ienny, F. Latourte, D. Lemosse, S. Pagano, E. Pagnacco, and F. Pierron. Overview of identification methods of mechanical parameters based on full-field measurements. Experimental Mechanics, 48:381-402, 2008.

[4] S. Belhabib, H. Haddadi, M. Gaspérini, and P. Vacher. Heterogeneous tensile test on elastoplastic metallic sheets: Comparaison between FEM simulations and fullfield strain measurements. International Journal of $\mathrm{Me}$ chanical Sciences, 50:14-21, 2007.

[5] M. Bornert, F. Brémand, P. Doumalin, M. Dupré, JC. Fazzini, M. Grédiac, F. Hild, S. Mistou, J. Molimard, J-J. Orteu, L. Robert, Y. Surrel, P Vacher, and B. Wattrisse. Assessment of digital image correlation measurement errors: Methodology and results. Experimental Mechanics, 49:353-370, 2008.

[6] D. Claire, S. Roux, and F. Hild. A finite element formulation to identify damage field: the equilibrium gap method. International Jounal of Numerical Methods in Engineering, 61:189-208, 2004.

[7] S. Cooreman, D. Lecompte, H. Sol, J. Vantonne, and D. Debruyne. Elasto-plastic material parameter identification by inverse methods: Calculation of the sensitivity 


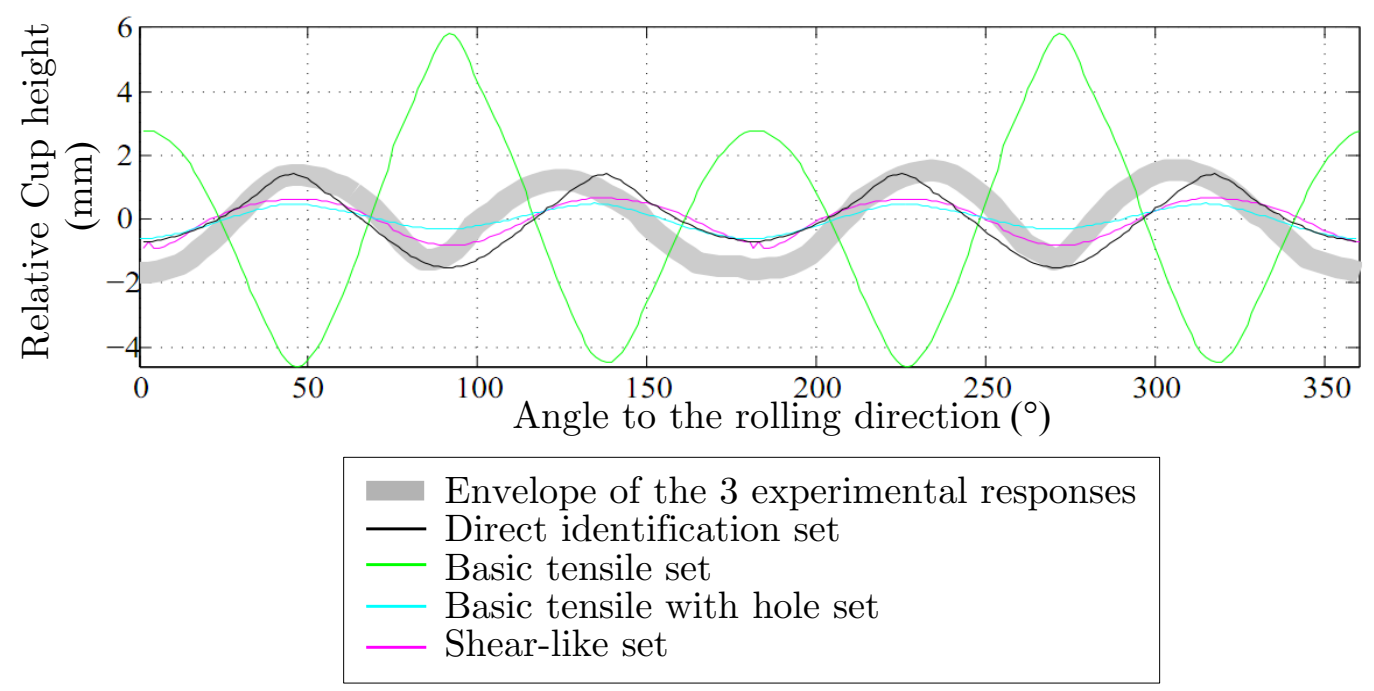

Figure 10: Comparison of predicted anisotropic profiles.

matrix. International Journal of Solids and Structures, 44:4329-4341, 2007.

[8] T. Coudert. Reconstruction tridimensionnelle du volume intérieur d'une chaussure : évaluation du chaussant. $\mathrm{PhD}$ thesis, Université de Savoie, 2005.

[9] L. Crouzeix, J-N. Périé, F. Collombet, and B. Douchin. An orthotropic variant of the equilibrium gap method applied to the analysis of a biaxial test on a composite material. Composites: Part A, 40:1732-1740, 2009.

[10] R. Fedele, B. Raka, F. Hild, and S. Roux. Identification of adhesive properties in glare assemblies by digital image correlation. J. Mech. Phys. Solids, 57:1003-1016, 2009.

[11] J.C. Gelin, O. Ghouati, and P. Paquier. Modelling and control of hydroforming processes for flanges forming. Annals of the CIRP, 47(1):213-216, 1998.

[12] O. Ghouati and J.C. Gelin. Identification of material parameters directly from metal forming processes. Journal of Material Processing Technology, 80-81:560-564, 1998.

[13] O. Ghouati and J.C. Gelin. A finite element-based identification method for complex metallic material behaviours. Computational Materials Science, 21:57-68, 2001.

[14] M. Grédiac. Principe des travaux virtuels et identification/principle of virtual work and identification. Comptes Rendus de l'Académie des Sciences., 309:1-5, 1989.

[15] M. Grédiac. The use of full-field measurement methods in composite material characterization: interest and limitations. Composites Part A: Applied Science and Manufacturing, 35:751-761, 2004.

[16] Z. Gronostajski. The constitutive equations for FEM analysis. Journal of Material Processing Technology, 106:40-44, 2000.

[17] C. G'Sell, J.M. Hiver, and A. Dahoun. Experimental characterization of deformation damage in solid polymers under tension, and its interrelation with necking.
International Journal of Solids and Structures, 39:3857$3872,2002$.

[18] T. Harth, S. Schwan, J. Lehn, and F.G. Kollmann. Identification of material parameters for inelastic constitutive models: statistical analysis and design of experiments. International Journal of Plasticity, 20:1403-1440, 2004.

[19] R. Hill. A theory of the yielding and plastic flow of anisotropic metals. Proc. Roy. Soc. London Ser. A, 193:281-297, 1948.

[20] R. Hill. A user-friendly theory of orthotropic plasticity in sheet metals. International Journal of Mechanical Sciences, 35:19-25, 1993.

[21] M . Ikehata. Inversion formulas for the linearized problem for an inverse boundary value problem in elastic prospection. SIAM Journal for Applied Mathematics, 50:1635-1644, 1990.

[22] J. Kajberg and G. Lindkvist. Characterization of materials subjected to large strains by inverse modeling based on in-plane displacement fields. International Journal of Solids and Structures, 41:3439-3459, 2004.

[23] K.T. Kavanagh and R.W. Clough. Finite element applications in the characterization of elastic solids. International Journal of Solids and Structures, 7:11-23, 1971.

[24] A. Khalfallah, H. Bel Hadj Salah, and A. Dogui. Anisotropic parameter identification using inhomogeneous tensile test. European Journal of Mechanics A/Solids, 21:927-942, 2002.

[25] P. Ladevèze, N. Moës, and B. Douchin. Constitutive relation error estimators for (visco)plastic finite element analysis with softening. Computer Methods in Applied Mechanics Engineering., 176:247-264, 1999.

[26] F. Latourte, A. Chrysochoos, S. Pagano, and B. Wattrisse. Elastoplastic behavior identification for heterogeneous loadings and materials. Experimental Mechanics, 48:435-449, 2008. 
[27] A Le Port, F Toussaint, and R Arrieux. Finite element study and sensitivity analysis of the deep-drawing formability of commercially pure titanium. International Journal of Material Forming, 2:121-129, 2009.

[28] D. Lecompte. Elastic and elasto-plastic material parameter identification by inverse modeling of static tests using digital image correlation. PhD thesis, Koninklijke Militaire School, Belgique, 2007.

[29] D. Lecompte, A. Smits, H. Sol, J. Vantomme, and D. Van Hemelrijck. Mixed numerical-experimental technique for orthotropic parameter identification using biaxial tensile test on cruciform specimens. International Journal of Solids and Structures, 44:1643-1656, 2007.

[30] K. Levenberg. A method for the solution of certain nonlinear problems in least-squares. Quart. Appl. Math., 2:164-168, 1944.

[31] C. Ma and L. Jiang. Some research on LevenbergMarquardt method for nonlinear equations. Applied Mathematics and Computation, 184:1032-1040, 2007.

[32] R. Mahnken. Aspects on the finite-element implementation of the Gurson model including parameter identification. International Journal of Plasticity, 15:1111-1137, 1999 .

[33] D.W. Marquardt. An algorithm for least-squares estimation of nonlinear parameters. J. Soc. Indust. Appl. Math., 11:431-441, 1963.

[34] M.H.H. Meuwissen. An inverse method for mechanical characterization of metal. $\mathrm{PhD}$ thesis, Eindhoven University of Technology, Netherlands, 1998.

[35] J. Molimard, R. Le Riche, A. Vautrin, and J.R. Lee. Identification of the four orthotropic plate stiffnesses using a single open-hole tensile test. Experimental Mechanics, 45:404-411, 2005.

[36] T. Nakamura and Y. Liu. Determination of nonlinear properties of thermal sprayed ceramic coatings via inverse analysis. International Journal of Solids and Structures, 44:1990-2009, 2007.
[37] B. Pan, K. Qian, H. Xie, and A. Asundi. Twodimensional digital image correlation for in-plane displacement and strain measurement: a review. Measurement science and technology, 20:1-17, 2009.

[38] J.-P. Ponthod and J.-P. Kleinermann. A cascade optimization methodology for automatic parameter identification and shape/process optimization in metal forming. Comput. Methods Appl. Mech. Engrg., 195:5472-5508, 2006.

[39] N. Promma, B. Raka, M. Grédiac, E. Toussaint, J.-B. LeCam, X. Balandraud, and F. Hild. Application of the virtual fields method to mechanical characterization of elastomeric materials. International Journal of Solids and Structures, 46:698-715, 2009.

[40] V. Tarigopula, O.S. Hopperstad, M. Langseth, A.H. Clausen, and F. Hild. A study of localisation in dualphase high-strength under dynamic loading using digital correlation and $\mathrm{FE}$ analysis. International Journal of Solids and Structures, 45:601-619, 2008.

[41] D.A. Tortorelli and P. Michaleris. Design sensitivity analysis: Overview and review. Inverse Problems in Engineering, 1:71-105, 1994.

[42] F. Toussaint, L. Tabourot, and F. Ducher. Experimental and numerical analysis of the forming process of $\mathrm{CP}$ titanium scoliotic instrumentation. Journal of Material Processing Technology, 197:10-16, 2008.

[43] P. Vacher, S. Dumoulin, F. Morestin, and S. MguilTouchal. Bidimensional strain measurement using digital images. Proc. Inst. Mech. Eng., 213:811-817, 1999.

[44] J-F. Witz, S. Roux, and F. Hild. Mechanical properties of crimped mineral wools : Identification from digital image correlation. Journal of Engineering Materials and Technology, 130:1-7, 2008.

[45] F. Yoshida, M. Urabe, and V.V. Toropov. Identification of material parameters in constitutive model for sheet metals from cyclic bending tests. International Journal of Mechanical Sciences, 40:237-249, 1998. 